\title{
Golden SPIRALS IN THE WAKE OF SEAL WhiskeR-INSPIRED ElLIPTIC CYLINDER PAIRS
}

c

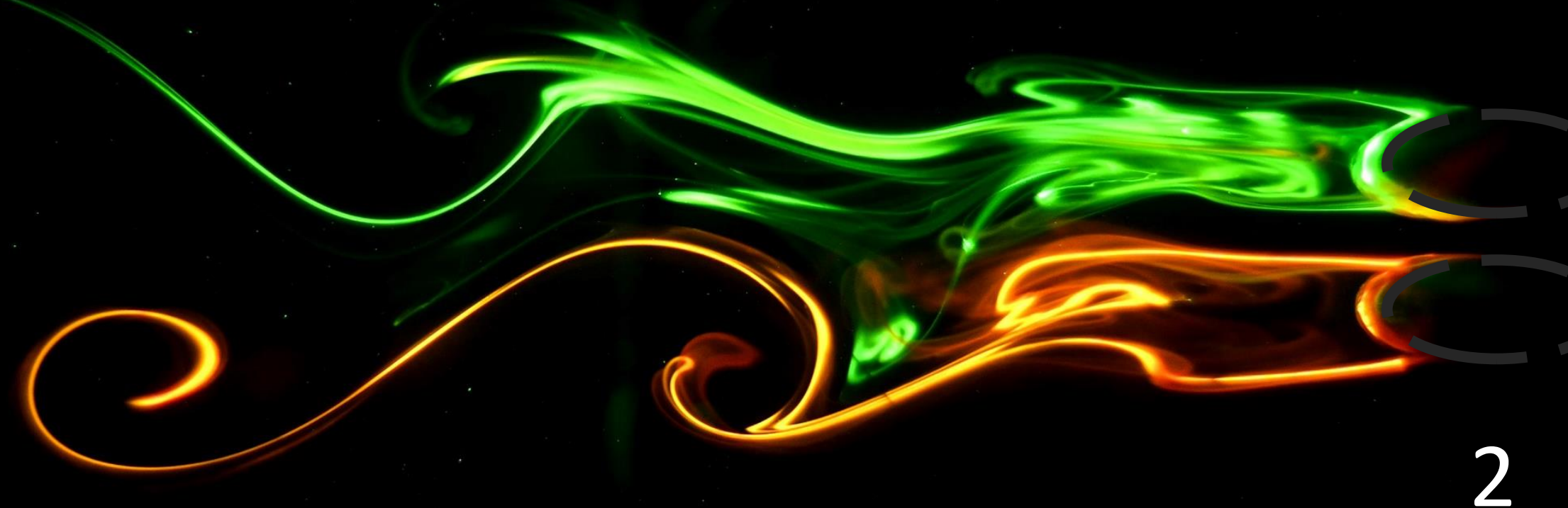

Seals have highly sensitive whiskers which they use to maneuver and find food by sensing the hydrodynamic trails left by prey. Unlike most mammals, the whiskers of seals have elliptical cross-sections with corrugations along the whisker axis. Recently, studies have investigated the wake generated behind single elliptic cylinders. However, since whiskers always develop as clusters - and can even have two or more whiskers emanating from the same follicle (Fig. 1 inset) - it is also important to understand the wake interactions that occur between multiple whiskers. Furthermore, the gap between two whiskers varies across their length, being smaller near the root and larger at the tips. To understand these effects, we investigate the wake interactions behind pairs of straight elliptic cylinders with different gap ratios, Reynolds number (Re) and elliptic eccentricity (e).

Pairs of ellipses are 3D printed using a stereolithography printer. These ellipses are mounted in a water tunnel. The vortex wake that forms behind each pair is visualized by painting the two ellipses with fluorescein dye and rhodamine dye prior to the experiment and illuminating the flow with a continuous 532nm laser.

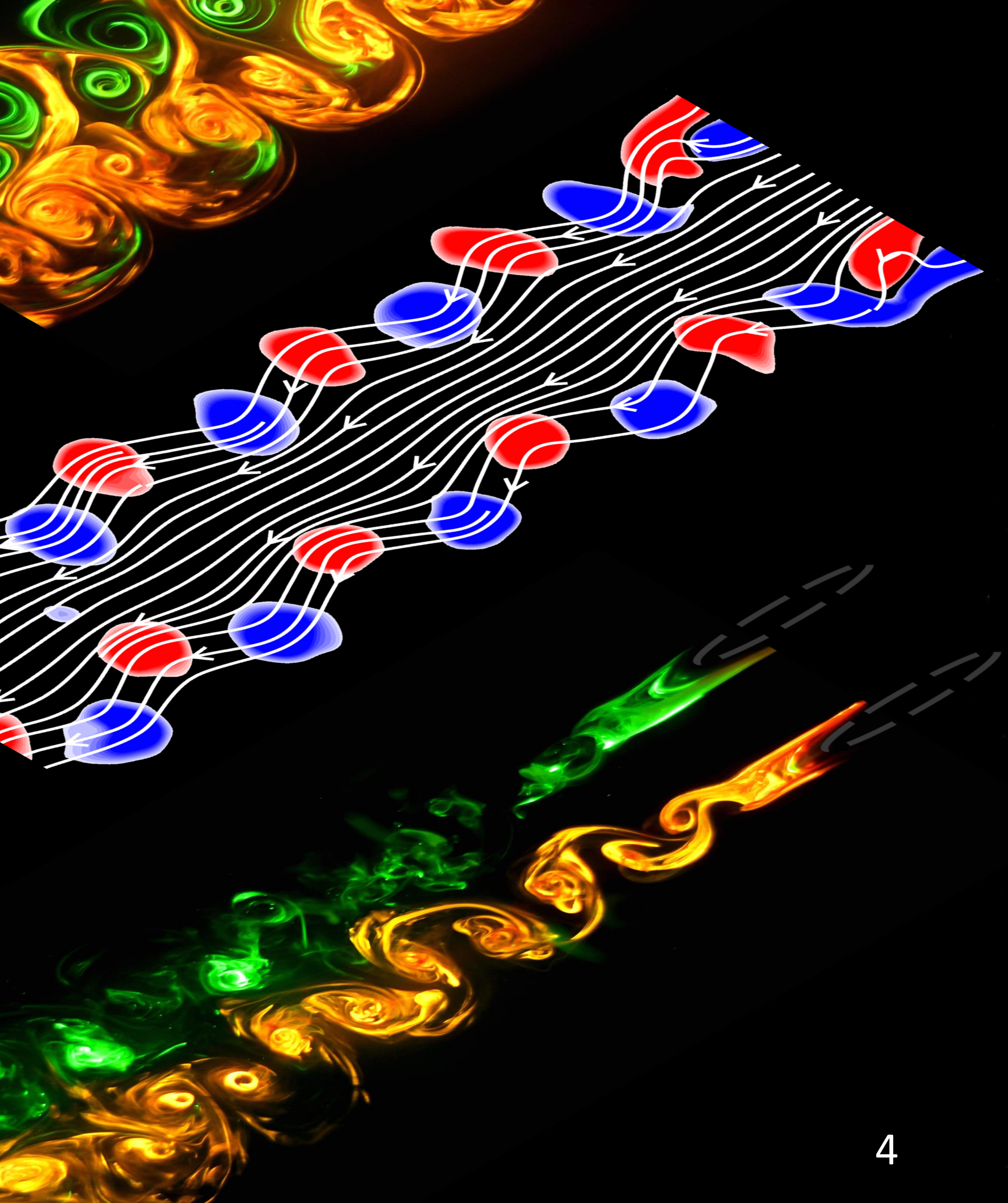

Applied Fluids Research Group, Auburn University
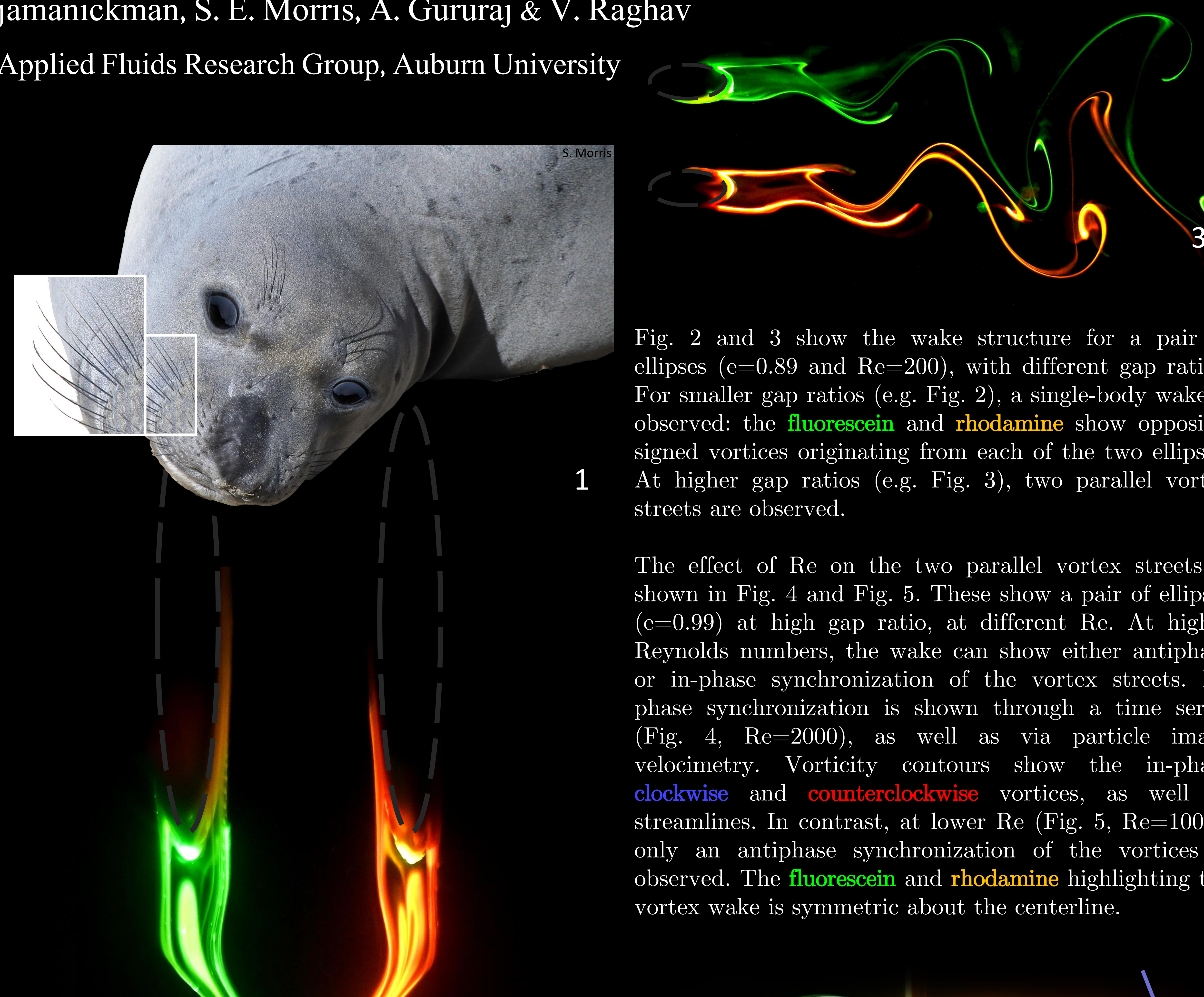

Fig. 2 and 3 show the wake structure for a pair of ellipses ( $\mathrm{e}=0.89$ and $\mathrm{Re}=200$ ), with different gap ratios. For smaller gap ratios (e.g. Fig. 2), a single-body wake is observed: the fluorescein and rhodamine show oppositesigned vortices originating from each of the two ellipses.

1 At higher gap ratios (e.g. Fig. 3), two parallel vortex streets are observed.

The effect of Re on the two parallel vortex streets is shown in Fig. 4 and Fig. 5. These show a pair of ellipses $(\mathrm{e}=0.99)$ at high gap ratio, at different Re. At higher Reynolds numbers, the wake can show either antiphase or in-phase synchronization of the vortex streets. Inphase synchronization is shown through a time series (Fig. 4, Re=2000), as well as via particle image velocimetry. Vorticity contours show the in-phase clockwise and counterclockwise vortices, as well as streamlines. In contrast, at lower Re (Fig. $5, \operatorname{Re}=1000$ ), only an antiphase synchronization of the vortices is observed. The fluorescein and rhodamine highlighting the vortex wake is symmetric about the centerline.

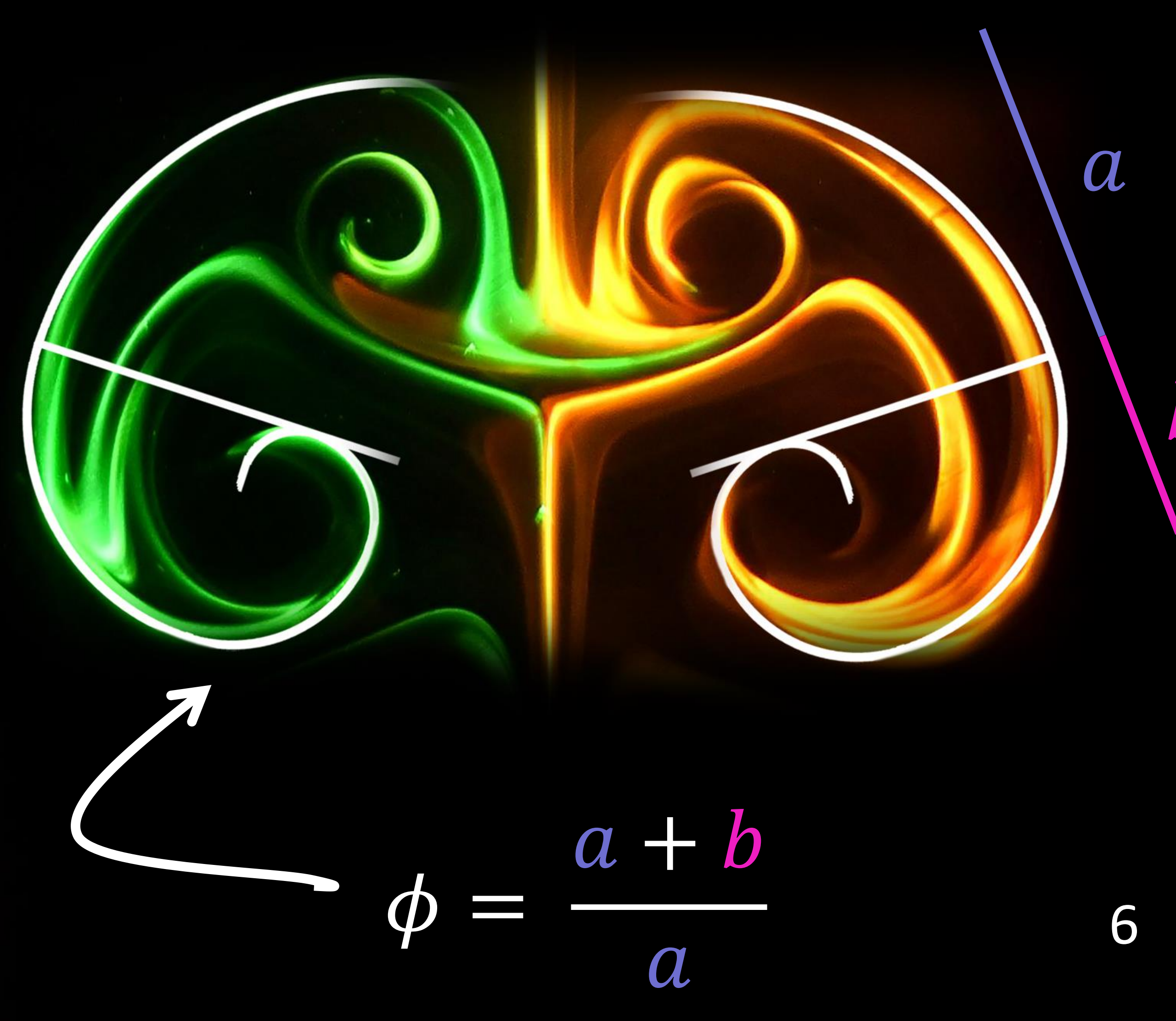

Interestingly, in the parameter space that leads to an anti-phase synchronization of the shed vortices in the parallel vortex streets regime, the rolling up of the two outer vortices yields 'golden spirals' (Fig. 5, 6). A golden spiral is one wherein the growth factor is equal to the golden ratio, $\phi=\frac{a+b}{a} \approx 1.62$. These golden spirals are famous both in nature and in art - from seashells and sunflowers, to Leonardo da Vinci's paintings of deluges and turbulence.

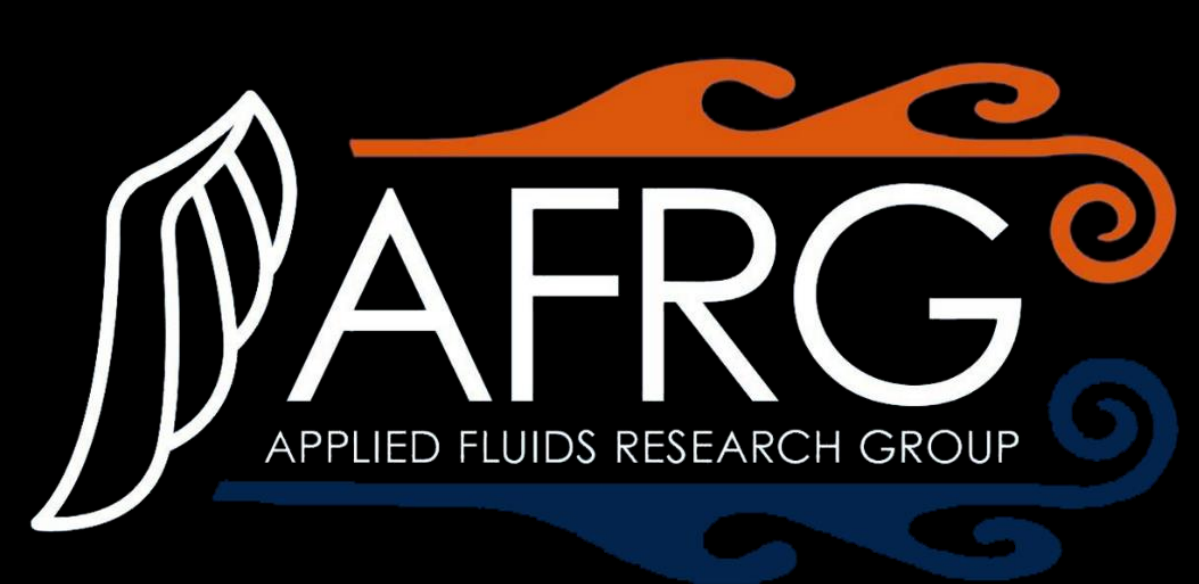

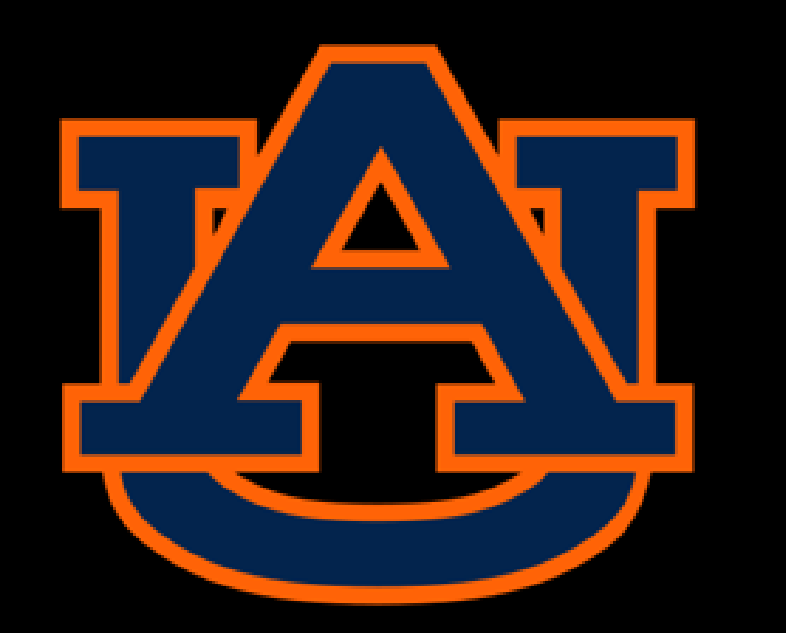

